\title{
Ellipsometric Selective Sensitivity to Magnetic Nanostructures
}

\author{
J. Hamrlová ${ }^{a, *}$, K. Postava ${ }^{a}$, O. ŽIvotský ${ }^{a}$, D. HrabovskÝ $^{a}$, J. Pištora ${ }^{a}$, P. Švec ${ }^{b}$, \\ D. JANIČKOVIČ ${ }^{b}$ AND A. MAZIEWSKI ${ }^{c}$ \\ ${ }^{a}$ Department of Physics, Technical University of Ostrava, 17. listopadu 15, Czech Republic \\ ${ }^{b}$ Institute of Physics, Slovak Academy of Sciences, Dúbravská cesta 9, Bratislava, Slovakia 15-424 \\ ${ }^{c}$ Laboratory of Magnetism, Institute of Physics, University of Białystok, Lipowa 41, 15-424 Białystok, Poland \\ Recently, we have shown that the approach of depth sensitivity of magneto-optic ellipsometry can be \\ generalized to selectivity from different materials in nanostructures. We use the condition number as the figure of \\ merit to quantify the magneto-optic selectivity to two different magnetic contributions in magnetic nanostructure. \\ The method is demonstrated on nanostructures containing magnetically hard Fe particles in surface layer of soft \\ FeNbB amorphous ribbon. We separated both magnetic contributions from measurement of hysteresis loops using \\ magneto-optic Kerr effect in longitudinal configuration. Magneto-optic selectivity is discussed and theoretical \\ model on the basis of effective medium is compared with experimental data of longitudinal magneto-optic Kerr \\ effect depending on angle of incidence.
}

PACS numbers: 78.20.Ls, 75.50.Bb, 75.60.-d

\section{Introduction}

Magnetic information included in the magneto-optic (MO) Kerr effect can be detected either using the Kerr rotation $\theta_{\mathrm{K}}$ or the Kerr ellipticity $\epsilon_{\mathrm{K}}$. Both quantities are represented by the complex MO Kerr effect $\boldsymbol{\Phi}_{\mathrm{K}}=$ $\theta_{\mathrm{K}}+\mathrm{i} \epsilon_{\mathrm{K}}$. When we have two magnetization contributions $m_{1}, m_{2}$ in material, then the total $\mathrm{MO}$ response is in very good approximation the sum of the contributions from particular magnetizations $\boldsymbol{\Phi}_{\text {tot }}=\boldsymbol{\Phi}_{1}+\boldsymbol{\Phi}_{2}$ [1]. The measured polar MO Kerr rotation $\theta_{\text {tot }}$ and ellipticity $\epsilon_{\text {tot }}$ can be expressed as weight sum of normalized particular magnetizations $m_{1}=M_{1} / M_{\mathrm{S} 1}, m_{2}=M_{2} / M_{\mathrm{S} 2}$, where $M_{\mathrm{S} 1,2}$ are corresponding saturated magnetizations [2]:

$$
\boldsymbol{\Phi}_{\mathrm{tot}}=\left[\begin{array}{c}
\theta_{\mathrm{tot}} \\
\epsilon_{\mathrm{tot}}
\end{array}\right]=\left[\begin{array}{ll}
a_{1} & a_{2} \\
b_{1} & b_{2}
\end{array}\right]\left[\begin{array}{l}
m_{1} \\
m_{2}
\end{array}\right]=\boldsymbol{A} \boldsymbol{M} .
$$

If we determine the elements of the matrix $\boldsymbol{A}$, we can obtain both magnetic contributions using the matrix inversion $\boldsymbol{M}=\boldsymbol{A}^{-1} \boldsymbol{\Phi}[2]$.

\section{Theory}

When we measure MO effect $\boldsymbol{\Phi}$ with the tolerance $\Delta \boldsymbol{\Phi}$, then nominal error of magnetic contribution is proportional to the condition number $\kappa(\boldsymbol{A})$ [3]:

$$
\begin{aligned}
& \frac{\|\Delta \boldsymbol{M}\|}{\|\boldsymbol{M}\|} \leq \kappa(\boldsymbol{A}) \frac{\|\Delta \Phi\|}{\|\Phi\|}, \\
& \kappa(\boldsymbol{A})=\left\|\boldsymbol{A}^{-1}\right\|\|\boldsymbol{A}\|,
\end{aligned}
$$

where $\|\boldsymbol{A}\|$ is the norm of the matrix $\boldsymbol{A}$. In our calculations we used the spectral norm [3].

\footnotetext{
* corresponding author; e-mail: jana.hamrlova@vsb.cz
}

To express the condition number analytically we express $\boldsymbol{\Phi}_{1}$ and $\boldsymbol{\Phi}_{2}$ as $\boldsymbol{\Phi}_{1}=\left|\boldsymbol{\Phi}_{1}\right| \exp \left(\mathrm{i} \alpha_{0}\right)$ and $\boldsymbol{\Phi}_{2}=$ $\left|\boldsymbol{\Phi}_{2}\right| \exp \left[\mathrm{i}\left(\alpha_{0}+\Delta \alpha\right)\right]$. This we can visualize in the $\theta \epsilon$ plane in Fig. 1a [4]. Then we can write matrix $\boldsymbol{A}$ :

$$
\boldsymbol{A}=\left|\boldsymbol{\Phi}_{1}\right|\left(\begin{array}{cc}
\cos \alpha_{0} & k \cos \left(\alpha_{0}+\Delta \alpha\right) \\
\sin \alpha_{0} & k \sin \left(\alpha_{0}+\Delta \alpha\right)
\end{array}\right) .
$$

Then the condition number is only function of angle $\Delta \alpha$ between vectors $\boldsymbol{\Phi}_{1}$ and $\boldsymbol{\Phi}_{2}$ and the ratio $k=\left|\boldsymbol{\Phi}_{2}\right| /\left|\boldsymbol{\Phi}_{1}\right|$ of absolute values of vectors $\left|\boldsymbol{\Phi}_{2}\right|,\left|\boldsymbol{\Phi}_{1}\right|$

$$
\begin{aligned}
& \kappa(\boldsymbol{A})=\frac{1}{2 k|\sin \Delta \alpha|} \\
& \quad \times\left(1+k^{2}+\sqrt{\left(k^{2}-1\right)^{2}+4 k^{2} \cos ^{2} \Delta \alpha}\right) .
\end{aligned}
$$

Figure $1 \mathrm{~b}$ shows dependence of the condition number $\kappa(\boldsymbol{A})$ from Eq. (5) on the parameters $\Delta \alpha$ and $k$. We see that we can obtain reasonable small condition number if parameter $k$ is not too different from 1 and vectors $\boldsymbol{\Phi}_{1}$ and $\boldsymbol{\Phi}_{2}$ are not collinear.

Then we can separate magnetization contributions of one material from two different depths $[5,6]$ but also magnetization contributions from two different materials $[2,7,8]$.

\section{Experimental results}

We can demonstrate this e.g. for magnetically hard $\mathrm{Fe}$ particles in surface layer of soft $\mathrm{FeNbB}$ amorphous ribbons [9]. On wheel side of $\mathrm{Fe}_{80.5} \mathrm{Nb}_{6.9} \mathrm{~B}_{12.6}$ ribbon $\mathrm{MO}$ hysteresis loops in longitudinal configuration were measured $[9,10]$. Similar loops measured at different angle of incidence $\left(65^{\circ}\right)$ are presented in Fig. 2a. The loops confirm that the near-surface region is inhomogeneous and contains contribution of different phases [10]. Transmission electron microscopy (TEM) measurements show the 


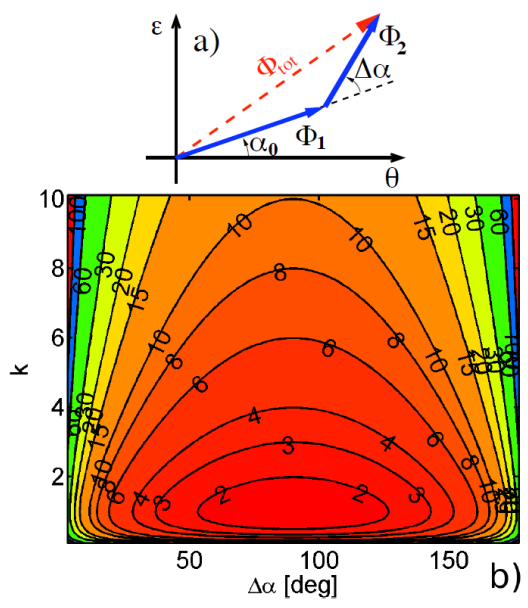

Fig. 1. (a) MO contributions $\boldsymbol{\Phi}_{1}, \boldsymbol{\Phi}_{2}$ to the total MO effect $\boldsymbol{\Phi}_{\text {tot }}$ displayed in $\theta, \epsilon$ plane. (b) Dependence of condition number $\kappa(\boldsymbol{A})$ of $2 \times 2$ matrix $\boldsymbol{A}$ of two column vectors $\boldsymbol{\Phi}_{1}, \boldsymbol{\Phi}_{2}$ on angle $\Delta \alpha$ between the vectors and parameter $k$ corresponding to the ratio of absolute values $\left|\boldsymbol{\Phi}_{1}\right|,\left|\boldsymbol{\Phi}_{2}\right|$. See Eq. (5).

$\alpha$-Fe crystallites with average size of grains about $100 \mathrm{~nm}$ situated near the wheel surface, Fig. 2b. By rotating of vector $\boldsymbol{\Phi}_{\text {tot }}$ with respect to the $\theta$ and $\epsilon$ axes we separated two different phases showed in Fig. 2c,d from measured loops in Fig. 2a. The corresponding matrix $\boldsymbol{A}$ and the condition number $\kappa(\boldsymbol{A})$ are

$$
\boldsymbol{A}_{\text {exp }}=\left(\begin{array}{cc}
-0.26 & -0.28 \\
-0.12 & 0.19
\end{array}\right), \quad \kappa\left(A_{\exp }\right)=1.8 .
$$

We suppose that the hard magnetic phase corresponds to the $\alpha$-Fe crystallites and the soft one corresponds to the amorphous FeNbB. The small value of the condition number $\kappa\left(\boldsymbol{A}_{\exp }\right)$ shows that the magnetic contributions are well separable.

Theoretically we describe the structure as spherical Fe particles with different size in amorphous bulk FeNbB. We calculated effective permittivity tensor using the Maxwell-Garnett approximation for anisotropic particles inserted in anisotropic medium [11]. For Fe particles we used optical and MO constants from $[12,13]$ and for bulk FeNbB the optical and MO constants from [9]. We supposed that the Fe volume fraction $f$ decreases exponentially with depth in amorphous material according to the function

$$
f=f_{0} \exp (-d / \Delta d) .
$$

On the surface we supposed an oxidation layer with refractive index $n=2.1$ (the refractive index of $\left.\mathrm{Nb}_{2} \mathrm{O}_{5}[14]\right)$.

Using Yeh's matrix formalism [15], we calculated contribution $\boldsymbol{\Phi}_{\text {tot }}$ and separately both magnetic contributions $\boldsymbol{\Phi}_{1}$ and $\boldsymbol{\Phi}_{2}$ to the longitudinal MO Kerr effect at wavelength of incident light $670 \mathrm{~nm}$ for angle of incidence varying from $0^{\circ}$ to $90^{\circ}$. We fitted parameters $f_{0}$, $\Delta d$ and thickness of surface oxidation layer $t_{\mathrm{s}}$ to the ex-

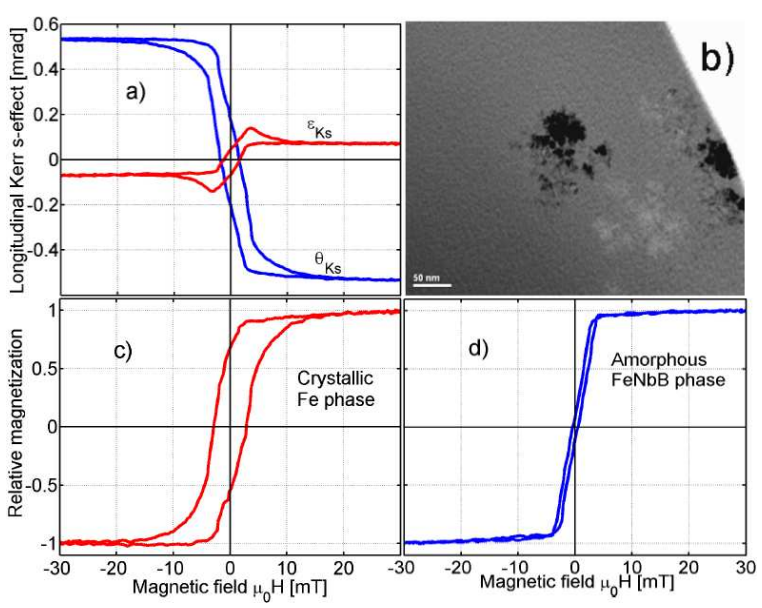

Fig. 2. (a) Sketch of the MO longitudinal Kerr rotation $\theta_{\mathrm{Ks}}$ and ellipticity $\epsilon_{\mathrm{Ks}}$ measured on wheel side of FeNbB ribbon for incident $s$-polarized wave at wavelength $670 \mathrm{~nm}$ and angle of incidence $65^{\circ}$. (b) Transmission electron microscopy on wheel side of the FeNbB ribbon. Dark and light areas show the crystallic $\alpha$-Fe phase. (c), (d) Hysteresis loops of the crystalline Fe phase and the amorphous FeNbB phase separated from data in (a), respectively.

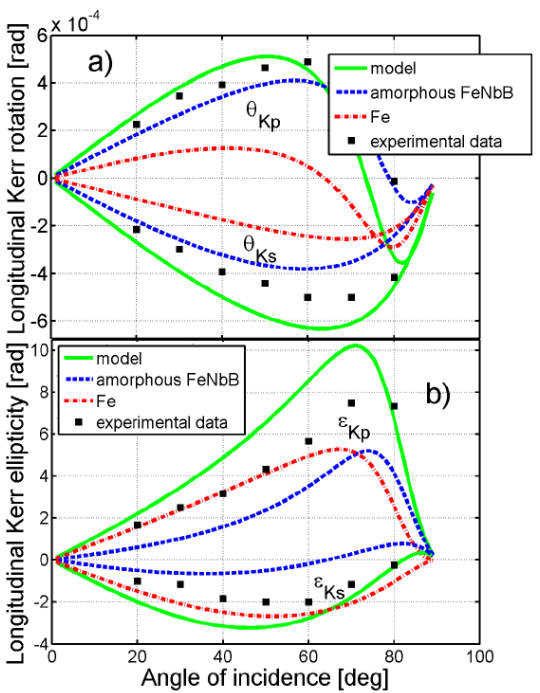

Fig. 3. Calculated dependence of longitudinal Kerr effect: (a) rotation, (b) ellipticity on angle of incidence for spherical Fe particles in homogeneous FeNbB material. The wavelength of incident light is $670 \mathrm{~nm}$. The contributions from $\mathrm{Fe}$ and from FeNbB are also shown separately. The model is compared with experimental data from [9] (square dots).

perimental data from [9] (Fig. 3). We obtained $f_{0}=0.8$, $\Delta d=7 \mathrm{~nm}$ and $t_{\mathrm{s}}=7 \mathrm{~nm}$. Further we confirmed that $\boldsymbol{\Phi}_{\text {tot }}=\boldsymbol{\Phi}_{1}+\boldsymbol{\Phi}_{2}$. For incident s-polarization wave at angle of incidence $65^{\circ}$ we calculated from $\boldsymbol{\Phi}_{1}$ and $\boldsymbol{\Phi}_{2}$ according to (1) matrix 


$$
\boldsymbol{A}_{\mathrm{eff}}=\left(\begin{array}{rr}
-0.25 & -0.37 \\
-0.23 & 0.004
\end{array}\right), \quad \kappa\left(\boldsymbol{A}_{\mathrm{eff}}\right)=2.5 .
$$

The matrix $\boldsymbol{A}_{\text {eff }}$ and corresponding condition number $\kappa\left(A_{\text {eff }}\right)$ obtained from model corresponds reasonably good with $\boldsymbol{A}_{\text {exp }}$ and $\kappa\left(\boldsymbol{A}_{\text {exp }}\right)$ (6) obtained from the experiment. The difference occurs because our model does not involve relatively big surface roughness, complicated shape of Fe crystallites and the fact that the structure of amorphous FeNbB bulk inside the ribbon can differ from the material filling space among Fe crystallites. Nevertheless, reasonably good agreement shows us that both contributions to the magnetization (from Fe particles and from amorphous FeNbB) are well separable and that we can use introduced model to describe the structure of wheel side of FeNbB ribbons.

\section{Acknowledgments}

Partial support from the projects $\mathrm{SP} / 2010150$, KAN 400100653, MSM6198910016, and CZ.1.05/2.1.00/ 01.0040 is acknowledged.

\section{References}

[1] R. Atkinson, P.H. Lissberger, J. Magn. Magn. Mater. 118, 271 (1993).

[2] K. Postava, I. Sveklo, M. Tekielak, P. Mazalski, A. Maziewski, A. Stupakiewicz, M. Urbaniak, B. Szymański, F. Stobiecki, IEEE Trans. Magn. 44, 3261 (2008).

[3] G. Strang, Introduction to Linear Algebra, Wellesley Cambridge Press, Wellesley, MA 2009.

[4] J. Hamrle, J. Ferré, M. Nývlt, Š. Višňovský, Phys. Rev. B 66, 224423 (2002).
[5] J. Ferré, P. Meyer, M. Nyvlt, S. Visnovsky, D. Renard, J. Magn. Magn. Mater. 165, 92 (1997).

[6] P. Vavassori, V. Bonanni, A. Busato, D. Bisero, G.G.A.O. Adeyeye, S. Goolaup, N. Singh, C. Spezzani, M. Sacchi, J. Phys. D, Appl. Phys. 41, 134014 (2008).

[7] K. Postava, D. Hrabovský, O. Životský, J. Pištora, N. Dix, R. Muralidharan, J.M. Caicedo, F. Sánchez, J. Fontcuberta, J. Appl. Phys. 105, 07C124 (2009).

[8] K. Postava, D. Hrabovský, J. Hamrlová, J. Pištora, A. Wawro, L.T. Baczewski, I. Sveklo, A. Maziewski, Thin Solid Films, to be published.

[9] O. Životský, K. Postava, K. Hrabovská, A. Hendrych, J. Pištora, L. Kraus, Appl. Surf. Sci. 255, 3322 (2008).

[10] O. Životský, L. Kraus, K. Postava, K. Barčová, J. Pištora, Y. Jirásková, J. Juraszek, J. Teillet, P. Švec, D. Janičkovič, J. Magn. Magn. Mater. 320, 1535 (2008).

[11] M. Abe, Phys. Rev. B 53, 7065 (1996).

[12] P.B. Johnson, R.W. Christy, Phys. Rev. B 9, 5056 (1974).

[13] Š. Višňovský, R. Krishnan, M. Nývlt, V. Prosser, J. Magn. Soc. Jpn. 20, 41 (1996).

[14] S. Venkataraj, R. Drese, O. Kappertz, R. Jayavel, M. Wuttig, Phys. Status Solidi A 188, 1047 (2001).

[15] Š. Višňovský, Czech. J. Phys. B 36, 625 (1986). 